\title{
Desempenho fotossintético, peroxidação lipídica e aspectos morfológicos de papuã exposto a diferentes condições hídricas do solo
}

\author{
Photosynthetic performance, lipid peroxidation and morphological \\ aspects of papua exposed to different soil water conditions
}

\author{
Leandro Lima Spatt' ${ }^{(\mathbb{D}}$, Sidinei José Lopes ${ }^{\prime \prime}\left(\mathbb{D}\right.$, Sylvio Henrique Bidel Dornelles ${ }^{\prime \prime \prime}(\mathbb{D}$, \\ Vinicius Severo Trivisiol ${ }^{\mathrm{IV}}(\mathbb{D})$ Mariane Peripolliv ${ }^{\mathrm{ID}}$, Elisandro Azeredo Nunes ${ }^{\mathrm{IV}}$ (i) \\ 'Coomat, Santa Maria, RS, Brasil \\ "Universidade Federal de Santa Maria, Dep. de Fitotecnia, Santa Maria,RS, Brasil \\ II' Universidade Federal de Santa Maria, Dep. De Biologia, Santa Maria, RS, Brasil \\ Iv Universidade Federal de Santa Maria, Santa Maria, RS, Brasil
}

\section{RESUMO}

O presente trabalho buscou elucidar o comportamento do biótipo de Urochloa plantaginea com características fisiológicas diferenciais, que permite um melhor crescimento e desenvolvimento em ambiente alagado. Utilizou-se o delineamento experimental inteiramente casualizado $2 \times 3$, sendo os fatores: populações de Urochloa plantaginea (Várzea e Coxilha) e condições hídricas do solo (50\% e 100\% da capacidade de retenção de água no solo (CRA); lâmina d'água de $5 \mathrm{~cm}$ ). Verificou-se as respostas relacionadas à parâmetros fotossintéticos e peroxidação lipídica $24 \mathrm{~h}$ e $192 \mathrm{~h}$ após o início das condições hídricas. Variáveis relacionadas à morfologia foram medidas no final do ciclo da planta. O biotipo de várzea frente ao biotipo de coxilha, demonstrou características morfofisiológicas superiores em condição de alagamento do solo. Pode-se ressaltar, maior eficiência no uso da água (cerca de 30\%), maior altura de plantas, menor peroxidação de lipídeos de parte aérea e um maior incremento de massa seca de parte aérea. Adicionalmente, o biotipo várzea demonstrou não variar os parâmetros fotossintéticos A, Gs, Ci, E, EUA e A/Ci quando exposta à lâmina d'água, isto comparando-se com a condição de $100 \%$ da CRA. As duas populações sobreviveram e encerraram seu ciclo produzindo sementes. Logo, evidenciase um possível processo adaptativo da população ao ambiente alagado.

Palavras-chave: Urochloa plantagine; Adaptação; Alagamento 


\section{ABSTRACT}

This paper aimed to elucidate the behavior of Urochloa plantaginea biotype with differential physiological characteristics, which allows a better growth and development in a flooded environment. A completely randomized experimental design was used $2 \times 3$, being the factors: populations of Urochloa plantaginea (lowlands and highlands) and soil water conditions (50\% and $100 \%$ of soil water retention capacity (CRA); water depth $5 \mathrm{~cm}$ ). Responses related to photosynthetic parameters and lipid peroxidation were verified $24 \mathrm{~h}$ and $192 \mathrm{~h}$ after the onset of water conditions. Morphology-related variables were measured at the end of the plant cycle. The lowland biotype compared to the highland biotype showed superior morphophysiological characteristics under soil flooding. It can be emphasized, higher water use efficiency (about 30\%), higher plant height, lower aerial part lipid peroxidation and higher aerial part dry matter increment. In addition, the lowland biotype was shown not to vary photosynthetic parameters A, Gs, Ci, E, USA and A / Ci when exposed to the water depth $5 \mathrm{~cm}$, compared with the 100\% CRA condition. Both populations survived and ended their cycle producing seeds. Thus, a possible adaptive process of the population to the flooded environment is evidenced.

Keywords: Urochloa plantaginea; Adaptation; Inundation

\section{INTRODUÇÃO}

O papuã (Urochloa plantaginea) é uma planta anual que se reproduz por semente, principalmente entre a primavera e o verão. Possui porte ereto e/ou semi-ereto (podendo atingir até 1 metro de altura) com intenso perfilhamento formando uma touceira ascendente (KISSMANN, 1997; SOUZA; MOREIRA; BRAGANÇA, 2010). As sementes têm baixa viabilidade logo após a maturação, porém passando o inverno, o poder germinativo aumenta, conservando-se por muitos anos. Segundo Kissmann (1997), tem-se conhecimento de áreas que voltaram a serem infestadas, após movimentação do solo, depois de 6 anos com cobertura por outra vegetação.

No Brasil, o papuã é uma das plantas daninhas mais frequentes nos solos cultivados das regiões Centro-Oeste e Sul e está presente em quase todos os Estados (LORENZI, 2000). Encontra-se em 62\% das áreas do Planalto do Rio Grande do Sul, sendo a gramínea com maior incidência nessa região (BIANCHI, 1996). Entretanto, esta espécie daninha tem ocorrido com bastante frequência em ambiente de terras baixas nas lavouras de arroz irrigado. Velho et al. (2012) constataram que 25 plantas $/ m^{2}$ de papuã em competição com o arroz ocasionaram um impacto negativo de $96 \%$ na produtividade da cultura. Anteriormente, esta 
espécie infestava as curvas de nível (taipas) na lavoura arrozeira, onde a condição de umidade do solo aproxima-se de $100 \%$ da capacidade de retenção de água. Porém, atualmente tornou-se comum o desenvolvimento desta planta no quadro sob lâmina d'água. Este fato pode estar ligado ao surgimento de um novo biótipo da espécie, na qual apresenta características fisiológicas e morfológicas que auxiliem na sua melhor adaptação a esta condição hídrica do solo.

As plantas pertencentes à família das Poaceae, caso da espécie em estudo, normalmente reagem à condições de baixa disponibilidade de oxigênio no solo através de alterações fisiológicas e morfológicas, como mudanças em rotas metabólicas, elongação da parte aérea, formação de raízes aéreas no colmo e aerênquimas (KRAEHMER; BAUR, 2013; WANG et al., 2014; MACEDO et al., 2015). Além disso, baixas concentrações de oxigênio atrelado à inundação do solo podem ocasionar queda na taxa fotossintética e fechamento estomático, prejudicando o desenvolvimento e acúmulo de matéria seca (ISMAIL et al., 2012; ARMSTRONG et al., 1994)

Levando em consideração os fatos explanados anteriormente, o presente trabalho buscou elucidar o surgimento de um novo biótipo de U. plantaginea com características fisiológicas diferenciais, na qual permita uma melhor adaptação e desenvolvimento em ambiente alagado. Para este fim, buscou-se determinar o comportamento fotossintético, a peroxidação de lipídeos e os parâmetros morfológicos de duas populações, sendo oriundas de terras altas (Coxilha) e baixas (Várzea) em diferentes condições hídricas do solo.

\section{MATERIAL E MÉTODOS}

O experimento foi realizado em casa de vegetação do Departamento de Biologia da Universidade Federal de Santa Maria - UFSM, Santa Maria, RS, com 6 m x 20 m e 5 m de pé direito, durante o período de outubro (2016) a março (2017). As amostras de Urochloa plantaginea foram coletadas em dois locais: 1) lavouras de 
arroz (Várzea) da cidade de Itaqui - Rio Grande do Sul (LAT: $29^{\circ} 15^{\prime}$ 6,2" S; LONG: $56^{\circ} 22^{\prime} 0,9^{\prime \prime}$ W); 2) em terras altas (Coxilha) de Augusto Pestana, planalto do Estado do Rio Grande do Sul (LAT: $28^{\circ} 28^{\prime}$ 17,2" S; LONG: 54 2' 7,8" W). A localização dos pontos de coleta foi aferida utilizando-se GPS, marca Garmin modelo Etrex 10, possibilitando assim retornar ao local de amostragem se necessário. Em uma área abrangendo 30 hectares, foram coletadas as sementes de plantas aleatórias pertencentes à população alvo, sendo as mesmas acondicionadas em sacos de papel poroso até o momento da semeadura. Para a correta identificação dos acessos coletados utilizou-se a chave de identificação das espécies da família Poaceae (BOLDRINI et al., 2008).

Utilizou-se o delineamento experimental inteiramente casualizado na forma de um bifatorial $2 \times 3$ com cinco repetições, sendo os fatores: A) duas populações de Urochloa plantaginea, sendo uma oriunda de área de Coxilha e outra de Várzea; B) três condições hídricas do solo: 50\%, 100\% da capacidade de retenção de água no solo (CRA) e lâmina d'água $(5 \mathrm{~cm})$. Para avaliar os parâmetros morfológicos utilizou-se vasos com a capacidade de 11 litros e 5 repetições, devido a necessidade de cultivar as plantas até o final do ciclo. Já para as variáveis relacionadas à fotossíntese foram utilizados vasos de 3 litros e 4 repetições. A determinação da CRA do solo foi realizada através da secagem até massa constante (estufa à $70^{\circ} \mathrm{C}$ ). Após a constatação de que a massa do solo atingiu constância, aferiu-se a capacidade de retenção de água do solo. No vaso a ser utilizado contendo uma massa de solo conhecida continha orifícios em sua base para a drenagem do excesso de água. O vaso foi irrigado até ocorrer a saturação do solo, sendo que, posteriormente, aguardou-se até o momento em que o mesmo atingiu massa constante. Assim, através da diferença de massa do vaso com solo seco para o vaso com solo úmido, obteve-se o valor da quantidade de água para atingir 100\% da capacidade de retenção de água. Para a determinação das condições hídricas do solo ( $50 \%$ e $100 \%$ da CRA) foram utilizadas as seguintes equações: 


$$
\begin{aligned}
& \text { MV50\% }=(\text { MVCRA }- \text { MVseco }) \times 0,5+\text { MVseco } \\
& \text { MV100\% }=(\text { MVCRA }- \text { MVseco }) \times 1,0+\text { MVseco }
\end{aligned}
$$

Em que: MVn\% é a massa do vaso para cada um dos tratamentos; MVCRA é a massa do vaso na capacidade de retenção de água; MVseco corresponde a massa do vaso preenchido com solo seco.

Recortes de tecido poroso foram utilizados para preencher os orifícios de drenagem na base do vaso, com o intuito de não ocorrer perda de solo no momento das irrigações. As irrigações foram realizadas diariamente, juntamente com a pesagem de cada vaso, utilizando uma balança eletrônica marca ACS System com precisão de $5 \mathrm{~g}$, sendo adicionado água até atingir a massa total prédeterminada (vaso + solo seco + volume de água para atingir 100\% e 50\% da CRA).

Para o correto estabelecimento das plantas, estas foram submetidas a uma condição de germinação de $100 \%$ da capacidade de retenção de água no solo. Anteriormente à semeadura, realizou-se um teste de germinação para determinar a viabilidade do lote de sementes coletadas e o número necessário de sementes por vaso. A partir deste resultado, onde se obteve uma média de $24 \%$ de germinação, optou-se por semear 10 propágulos por recipiente em vasos de $11 \mathrm{~L}$ e 30 sementes em vasos de 3 L. A variação no número de sementes por vaso foi adequada ao fim, devido a análise de peroxidação de lipídeos $(3 \mathrm{~L})$ ser destrutiva e necessitar de um mínimo de massa seca para a análise na qual uma planta não seria suficiente. Após a emergência ocorreu o raleio das plântulas, permanecendo uma planta por vaso de $11 \mathrm{~L}$ e 3 plantas por vaso de $3 \mathrm{~L}$. Os tratamentos referentes à condição hídrica do solo iniciaram 28 dias após a semeadura, quan do as plantas de U. plantaginea apresentavam de 3-4 folhas completamente desenvolvidas (simulando a condição de entrada d'água na lavoura de arroz irrigado).

As avaliações dos parâmetros relacionados à fotossíntese, foram aferidas no terço médio da última folha completamente expandida de cada planta. As plantas de papuã encontravam-se no estádio 3-4 folhas no momento da leitura dos dados, 
realizada 24 e 192 horas após o início dos tratamentos de condição hídrica do solo. O equipamento utilizado foi o medidor portátil Infra Red Gas Analyzer (IRGA) (modelo LI-6400 XT, marca LI-COR), utilizando uma radiação fotossintética de 1000 $\mu \mathrm{mol} \mathrm{m} \mathrm{s}^{-1}$ e concentração de $\mathrm{CO}_{2}$ de $400 \mathrm{~mol} \mathrm{~mol}^{-1}$. As variáveis determinadas foram: taxa fotossintética ( $\mathrm{A}-\mu \mathrm{mol} \mathrm{CO}_{2} \mathrm{~m}^{-2} \mathrm{~s}^{-1}$ ); condutância estomática de vapores de água (Gs - mol $\left.\mathrm{H}_{2} \mathrm{O} \mathrm{m}^{-2} \mathrm{~s}^{-1}\right)$; concentração interna de $\mathrm{CO}_{2}\left(\mathrm{Ci}-\mu \mathrm{mol} \mathrm{CO}_{2}\right.$ mol air1); taxa transpiratória ( $\mathrm{E}-\mathrm{mol} \mathrm{H}_{2} \mathrm{O} \mathrm{m}^{-2} \mathrm{~s}^{-1}$ ); a eficiência do uso da água ( $\mathrm{EUA}$ - mol $\mathrm{CO}_{2}$ mol $\mathrm{H}_{2} \mathrm{O}^{-1}$ ), obtida pela relação entre quantidade de $\mathrm{CO}^{2}$ fixado pela fotossíntese e quantidade de água transpirada e; a eficiência da carboxilação da enzima rubisco $(\mathrm{A} / \mathrm{Ci})$, obtida pela relação entre quantidade de $\mathrm{CO}_{2}$ fixado na fotossíntese e a concentração interna de $\mathrm{CO}_{2}$. As avaliações ocorreram no período das 8:00 às 10:00 horas da manhã, em dia ensolarado. Após a avaliação fotossintética nos respectivos períodos de 24 e 192 horas, foram realizadas a coleta de parte aérea e raiz com o intuito de estimar a peroxidação de lipídeos.

A peroxidação de lipídios foi avaliada pelo método de El-Moshaty et al. (1993), em que as amostras de raíz e parte aérea, logo após a coleta, foram envoltas por papel alumínio e congeladas em nitrogênio líquido contido em caixa de isopor. Após a coleta, as amostras ficaram armazenadas em um freezer com a temperatura de $-80{ }^{\circ} \mathrm{C}$ até o momento do início da técnica laboratorial para estimativa dos dados. Amostras frescas de raízes e parte aérea $(0,5 \mathrm{~g})$ foram maceradas em nitrogênio líquido e homogeneizadas em $5 \mathrm{~mL}$ de $0,2 \mathrm{M}$ de tampão citrato $(\mathrm{pH} 6,5)$ contendo $0,5 \%$ de Triton X-100. O homogeneizado foi centrifugado por $15 \mathrm{~min}$ a $20.000 \times \mathrm{g}$. Um mL do sobrenadante foi adicionado a $1 \mathrm{~mL}$ de TCA $20 \%$ $(\mathrm{w} / \mathrm{v})$ contendo $0,5 \%(\mathrm{w} / \mathrm{v})$ de ácido tiobarbitúrico. A mistura foi aquecida à $95^{\circ} \mathrm{C}$ por 40 min e então resfriada por 15 min, sendo centrifugada a $10.000 \times$ g por 15 min. A absorbância do sobrenadante foi lida a 532 e $600 \mathrm{~nm}$ (para corrigir a turbidez não específica). A peroxidação lipídica foi expressa em nmol MDA mg-1 de proteína. 
Ao final do ciclo foram realizadas avaliações das variáveis morfológicas MSR (massa seca de raiz), MSPA (massa seca de parte aérea), AFPM (altura final da planta mãe) e NTP (número total de perfilhos). Para as avaliações de massa seca, as plantas foram retiradas dos vasos, lavadas, e secas em estufa a temperatura de $70^{\circ} \mathrm{C}$ até massa constante.

A análise de variância para os dados foi realizada conforme o modelo matemático do delineamento inteiramente casualizado com arranjo bifatorial. Os erros experimentais foram testados quanto à normalidade de sua distribuição através do teste de Shapiro-Wilk e a homogeneidade das variâncias através do teste de Bartlett, com auxílio do programa Action (ESTATCAMP, 2014). Posteriormente, procedeu-se à análise de variância (ANOVA) e o teste de ScottKnott para agrupamento das médias, em $5 \%$ de probabilidade de erro $(p<0,05)$, utilizando-se o programa estatístico Sisvarß 5.3 (FERREIRA, 2011).

\section{RESULTADOS E DISCUSSÕES}

Verificou-se interação entre o fator condição hídrica do solo e população de Urochloa plantaginea para a variável condutância estomática (GS - mol de $\mathrm{H}_{2} \mathrm{O}$ m $\left.{ }^{2} \mathrm{~S}^{-1}\right)$, ocorrendo 192 horas após o início dos tratamentos de condição hídrica do solo (Tabela 1). Considerando-se a média geral das populações de Várzea e Coxilha, houve uma maior condutância estomática em condições de estresse hídrico, seja na falta d'água ( $50 \%$ da CRA do solo) ou excesso (Lâmina). Analisando-se as populações isoladamente pode-se concluir que a população Coxilha apresentou maior condutância estomática em condição de alagamento. Sendo que na população Várzea não houve diferença entre as três condições hídricas do solo, demonstrando comportamento semelhante nestas três condições. 
Tabela 1 - Médias da condutância estomática (GS - mol de $\mathrm{H}_{2} \mathrm{O} \mathrm{m}^{-2} \mathrm{~s}^{-1}$ ) da interação entre duas populações de Urochloa plantaginea e diferentes condições hídricas do solo 192 horas após o início dos tratamentos de umidade do solo e resumo do teste $\mathrm{F}$ da Análise de Variância

\begin{tabular}{lcccccccc}
\hline \multirow{2}{*}{ Condição hídrica do solo } & \multicolumn{9}{c}{ População } & \multirow{2}{*}{ Média } \\
\cline { 2 - 7 } & 0,12 & $\mathrm{y}$ & $\mathrm{B}$ & 0,19 & $\mathrm{~A}$ & $\mathrm{~A}$ & $0,15 \mathrm{~A}$ \\
\hline $50 \%$ CRA & 0,11 & $\mathrm{a}$ & $\mathrm{B}$ & 0,09 & $\mathrm{~A}$ & $\mathrm{~A}$ & $0,10 \mathrm{~B}$ \\
$100 \%$ CRA & 0,20 & $\mathrm{a}$ & $\mathrm{A}$ & 0,12 & $\mathrm{~B}$ & $\mathrm{~A}$ & $0,16 \mathrm{~A}$ \\
Lâmina d'água & 0,15 & $\mathrm{a}$ & & 0,13 & $\mathrm{~A}$ & & \\
Média & & & & &
\end{tabular}

CV (\%) 36,36

Resumo do teste de F da Análise de Variância
Teste de $F_{\text {população }}(A)$
$0,34 \mathrm{~ns}$
Teste de $F_{\text {condição hídrica }}(\mathrm{C})$
$3,12 \mathrm{~ns}$
Teste $\mathrm{F}$ da interação $\mathrm{A} \times \mathrm{C}$ $3,68 *$

Médias não seguida pela mesma letra, maiúscula na coluna e minúscula na linha, diferem entre si pelo teste de Scott-Knott, em 5\% de probabilidade. CRA: capacidade de retenção de água do solo. * significância em 5\%. ns: não significativo em nível de 5\% de probabilidade de erro.

Plantas adaptadas ou em processo de adaptação à condições de estresse hídrico, seja por falta ou excesso de água no solo, demonstram uma maior estabilidade na condutância estomática quando expostas a esta condição (MOLLARD et al., 2008). Na condição de Lâmina d'água as plantas de Coxilha obtiveram condutância estomática superior às de Várzea. Algumas espécies adaptadas ao ambiente alagado fecham seus estômatos quando expostas a condição de lâmina d'água (KOZLOWSKI, 1984). Este fato está intimamente ligado com a redução na condutância estomática e transpiração, assim, diminuindo a velocidade na absorção de água pelas raízes (PEZESHKI, 2001). Complementarmente, a redução na taxa de transpiração da planta em processo de adaptação pode estar atribuída a nova estruturação e diferenciação celular, formando aerênquimas, bloqueios no xilema e lignificação celular, assim reduzindo a condutividade hidráulica das raízes (BRAMLEY; TYERMAN, 2010; HERZOG et al., 2016). A formação de aerênquima lisígeno em Urochloa plantaginea em ambiente alagado já foi caracterizada por Macedo (2015). 
$\mathrm{Na}$ avaliação 24 horas após o início dos tratamentos de condição hídrica do solo, os parâmetros taxa fotossintética ( $\mathrm{A}-\mu \mathrm{mol}$ de $\left.\mathrm{CO}_{2} \mathrm{~m}^{-2} \mathrm{~s}^{-1}\right)$, condutância estomática de vapores de água ( $\mathrm{Gs}-\mathrm{mol}$ de $\mathrm{H}_{2} \mathrm{O} \mathrm{m}^{-2} \mathrm{~s}^{-1}$ ), concentração de $\mathrm{CO}_{2}$

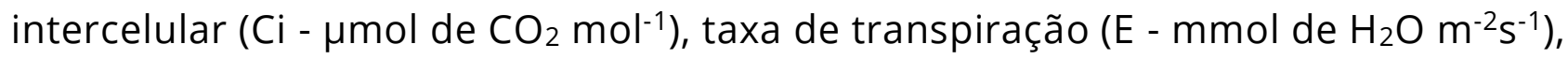
eficiência do uso da água (EUA - mol de $\mathrm{CO}_{2} \mathrm{~mol} \mathrm{H}_{2} \mathrm{O}^{-1}$ ) e eficiência instantânea de carboxilação pela rubisco ( $\left.\mathrm{A} / \mathrm{Ci} \mu \mathrm{mol} \mathrm{m}^{-2} \mathrm{~s}^{-1} \mathrm{~Pa}^{-1}\right)$ não apresentaram interação significativa entre os fatores condição hídrica do solo e população (Tabela 2). Da mesma maneira, as aferições realizadas 192 horas após o início dos tratamentos de condição hídrica do solo as variáveis $A, C i, E$, EUA e $A / C i$ não apresentaram interação entre os fatores estudados.

Tabela 2 - Médias da taxa fotossintética ( $\mathrm{A}$ - $\mu \mathrm{mol}$ de $\mathrm{CO}_{2} \mathrm{~m}^{-2} \mathrm{~s}^{-1}$ ), condutância estomática de vapores de água (Gs - mol de $\mathrm{H}_{2} \mathrm{O} \mathrm{m} \mathrm{m}^{-2} \mathrm{~s}^{-1}$ ) e concentração de $\mathrm{CO}_{2}$ intercelular $\left(\mathrm{Ci}-\mu \mathrm{mol}\right.$ de $\mathrm{CO}_{2} \mathrm{~mol}^{-1}$ em duas populações de plantas de Urochloa plantaginea 24 horas após o início dos tratamentos de condições hídricas do solo e resumo do teste de F da Análise de Variância

\begin{tabular}{|c|c|c|c|c|c|c|c|}
\hline \multirow{3}{*}{ Condição Hídrica } & \multicolumn{2}{|c|}{ A } & \multicolumn{3}{|c|}{ Gs } & \multicolumn{2}{|c|}{$\mathbf{C i}$} \\
\hline & \multicolumn{7}{|c|}{ População } \\
\hline & $\operatorname{cox}$ & VAR & $\operatorname{cox}$ & VAR & Média & $\operatorname{cox}$ & VAR \\
\hline $50 \% C R A$ & $16,45 \mathrm{~B} \mathrm{a}$ & $25,65 \mathrm{~A} \mathrm{a}$ & $0,13 \mathrm{~B} \mathrm{a}$ & $0,16 \mathrm{~B} \mathrm{a}$ & $0,14 \mathrm{~B}$ & $187,59 \mathrm{~A} \mathrm{a}$ & 97,66 A b \\
\hline $100 \% C R A$ & $14,07 \mathrm{~B} \mathrm{a}$ & $17,78 \mathrm{~A} a$ & $0,11 \mathrm{~B} \mathrm{a}$ & $0,12 \mathrm{~B} \mathrm{a}$ & $0,12 \mathrm{~B}$ & $182,83 \mathrm{~A} \mathrm{a}$ & $147,57 \mathrm{~A} \mathrm{a}$ \\
\hline$L A M$ & $33,04 \mathrm{~A} a$ & $32,05 \mathrm{~A}$ a & $0,29 \mathrm{~A} a$ & $0,27 \mathrm{~A} \mathrm{a}$ & $0,28 \mathrm{~A}$ & $164,62 \mathrm{~A} a$ & $152,39 \mathrm{~A} \mathrm{a}$ \\
\hline MÉDIA & - & - & - & - & - & $178,34 \mathrm{a}$ & $132,53 \mathrm{~b}$ \\
\hline CV (\%) & \multicolumn{2}{|c|}{41,13} & \multicolumn{3}{|c|}{44,19} & \multicolumn{2}{|c|}{26,81} \\
\hline \multicolumn{8}{|c|}{ Resumo do teste de F da Análise de Variância } \\
\hline$F_{\text {população }}(A)$ & \multicolumn{2}{|c|}{$1,05 \mathrm{~ns}$} & \multicolumn{3}{|c|}{$0,02 \mathrm{~ns}$} & \multicolumn{2}{|c|}{$7,25 *$} \\
\hline $\mathrm{F}_{\text {cond.híd. }}(\mathrm{C})$ & \multicolumn{2}{|c|}{$6,39 *$} & \multicolumn{3}{|c|}{$9,24 *$} & \multicolumn{2}{|c|}{0,61 ns } \\
\hline$F(A) \times F(C)$ & \multicolumn{2}{|c|}{$0,57 \mathrm{~ns}$} & \multicolumn{3}{|c|}{$0,20 \mathrm{~ns}$} & \multicolumn{2}{|c|}{1,83 ns } \\
\hline
\end{tabular}

Médias não seguida pela mesma letra, maiúscula na coluna e minúscula na linha, diferem entre si pelo teste de Scott-Knott, em 5\% de probabilidade. COX: Coxilha; VAR: Várzea. CRA: capacidade de retenção de água do solo; LAM: Lâmina d'água. * significância em 5\%. ns: não significativo em nível de 5\% de probabilidade de erro. 
As populações Coxilha e Várzea evidenciaram comportamentos diferenciados quanto à eficiência no uso da água (EUA) nos dois momentos avaliados (24 horas e 192 horas). Em média, a população de papuã oriunda de um ambiente de Várzea, caracterizou-se por ser mais eficiente no uso da água perante as plantas oriundas de terras altas (Coxilha) (Tabela 3).

Tabela 3 - Médias da taxa de transpiração ( $E$ - mmol de $\mathrm{H}_{2} \mathrm{O} \mathrm{m} \mathrm{m}^{-2} \mathrm{~s}^{-1}$ ), eficiência do uso da água (EUA - mol de $\mathrm{CO}_{2} \mathrm{~mol} \mathrm{H}_{2} \mathrm{O}^{-1}$ ) e eficiência instantânea de carboxilação pela rubisco ( $\left.\mathrm{A} / \mathrm{Ci}-\mu \mathrm{mol} \mathrm{m} \mathrm{m}^{-2} \mathrm{~S}^{-1} \mathrm{~Pa}^{-1}\right)$ em duas populações de plantas de Urochloa plantaginea 24 horas após o início dos tratamentos de condições hídricas do solo e resumo do teste de F da Análise de Variância

\begin{tabular}{|c|c|c|c|c|c|c|c|c|c|}
\hline \multirow{3}{*}{ Condição Hídrica } & \multicolumn{3}{|c|}{$\mathbf{E}$} & \multicolumn{3}{|c|}{ EUA } & \multicolumn{3}{|c|}{$\mathrm{A} / \mathrm{Ci}$} \\
\hline & \multicolumn{9}{|c|}{ População } \\
\hline & $\operatorname{cox}$ & VAR & Média & $\operatorname{cox}$ & VAR & Média & $\operatorname{cox}$ & VAR & Média \\
\hline $50 \% C R A$ & $2,44 \mathrm{~B} \mathrm{a}$ & $2,70 \mathrm{~B} \mathrm{a}$ & $2,57 \mathrm{~B}$ & $6,08 \mathrm{~A} \mathrm{~b}$ & $9,63 \mathrm{~A} a$ & $7,86 \mathrm{~A}$ & $0,12 \mathrm{~A} \mathrm{~b}$ & $0,29 \mathrm{~A} \mathrm{a}$ & $0,20 \mathrm{~A}$ \\
\hline $100 \% C R A$ & $2,50 \mathrm{~B} \mathrm{a}$ & $2,50 \mathrm{~B} \mathrm{a}$ & $2,50 \mathrm{~B}$ & $5,52 \mathrm{~A} a$ & 7,04 B a & $6,28 \mathrm{~B}$ & $0,08 \mathrm{~A} a$ & $0,12 \mathrm{~A} \mathrm{a}$ & $0,10 \mathrm{~B}$ \\
\hline$L A M$ & $5,63 \mathrm{~A} \mathrm{a}$ & $5,02 \mathrm{~A} a$ & $5,33 \mathrm{~A}$ & $5,80 \mathrm{~A} \mathrm{a}$ & $6,37 \mathrm{~B} \mathrm{a}$ & $6,08 \mathrm{~B}$ & $0,20 \mathrm{~A} \mathrm{a}$ & $0,21 \mathrm{~A} \mathrm{a}$ & $0,20 \mathrm{~A}$ \\
\hline Média & - & - & - & $5,80 \mathrm{~b}$ & $7,68 \mathrm{a}$ & - & - & - & - \\
\hline \multirow[t]{2}{*}{ CV (\%) } & \multicolumn{3}{|c|}{35,43} & \multicolumn{3}{|c|}{19,94} & \multicolumn{3}{|c|}{52,73} \\
\hline & \multirow{2}{*}{\multicolumn{6}{|c|}{ Resumo do teste de F da Análise de Variância }} & & & \\
\hline $\mathrm{F}_{\text {população }}(\mathrm{A})$ & & & & & & & \multicolumn{3}{|c|}{$3,59 \mathrm{~ns}$} \\
\hline F cond.híd. (C) & \multicolumn{3}{|c|}{$13,72^{\star}$} & \multicolumn{3}{|c|}{$\begin{array}{l}11,76^{*} \\
4,18^{*}\end{array}$} & \multicolumn{3}{|c|}{$3,24 *$} \\
\hline$F(A) \times F(C)$ & \multicolumn{3}{|c|}{$0,26 \mathrm{~ns}$} & \multicolumn{3}{|c|}{$2,57 \mathrm{~ns}$} & \multicolumn{3}{|c|}{$1,78 \mathrm{~ns}$} \\
\hline
\end{tabular}

Em contrapartida, a população de Coxilha diferenciou-se por apresentar maior Ci comparando-se com as plantas de Várzea na primeira avaliação. Porém, esta diferença na Ci não se confirmou na avaliação de 192 horas após o início dos tratamentos (Tabela 4). Para as variáveis A, Gs, E e A/Ci não foi possível comprovar 
a existência de diferenças entre as populações avaliadas no presente estudo, considerando as médias gerais dos tratamentos de condição hídrica do solo.

Tabela 4 - Médias da taxa fotossintética $\left(\mathrm{A}\right.$ - $\mu \mathrm{mol}$ de $\mathrm{CO}_{2} \mathrm{~m}^{-2} \mathrm{~s}^{-1}$ ), concentração de $\mathrm{CO}_{2}$ intercelular ( $\mathrm{Ci}$ - $\mu \mathrm{mol}$ de $\mathrm{CO}_{2} \mathrm{~mol}^{-1}$ ) e taxa de transpiração ( $\mathrm{E}-\mathrm{mmol}$ de $\mathrm{H}_{2} \mathrm{O} \mathrm{m}^{-2} \mathrm{~s}^{-1}$ ) em duas populações de plantas de Urochloa plantaginea, 192 horas após o início dos tratamentos de condições hídricas do solo e resumo do teste de $\mathrm{F}$ da Análise de Variância

\begin{tabular}{|c|c|c|c|c|c|c|c|c|}
\hline \multirow{3}{*}{ Condição Hídrica } & \multicolumn{2}{|c|}{ A } & \multicolumn{3}{|c|}{$\mathbf{C i}$} & \multicolumn{3}{|c|}{$\mathbf{E}$} \\
\hline & \multicolumn{8}{|c|}{ População } \\
\hline & $\operatorname{cox}$ & VAR & $\operatorname{cox}$ & VAR & Média & $\operatorname{cox}$ & VAR & Média \\
\hline $50 \% C R A$ & $16,72 \mathrm{~ns}^{1}$ & $21,59 \mathrm{~ns}$ & $165,17 \mathrm{Aa}$ & $183,96 \mathrm{~A} \mathrm{a}$ & $174,56 \mathrm{~A}$ & $2,55 \mathrm{~B} \mathrm{a}$ & $3,10 \mathrm{~A} \mathrm{a}$ & $2,83 \mathrm{~B}$ \\
\hline $100 \% C R A$ & 18,49 & 16,12 & $113,82 \mathrm{~B} \mathrm{a}$ & $106,03 \mathrm{~B} \mathrm{a}$ & 109,92 B & $2,60 \mathrm{~B} \mathrm{a}$ & $2,12 \mathrm{~A} \mathrm{a}$ & $2,36 \mathrm{~B}$ \\
\hline$L A M$ & 22,55 & 19,74 & $177,22 \mathrm{~A} a$ & $118,76 \mathrm{~B} \mathrm{~b}$ & $147,99 \mathrm{~A}$ & $4,35 \mathrm{~A} \mathrm{a}$ & $2,91 \mathrm{~A} \mathrm{~b}$ & $3,63 \mathrm{~A}$ \\
\hline$\overline{C V}(\%)$ & 31, & 79 & & 22,61 & & & 27,64 & \\
\hline \multicolumn{9}{|c|}{ Resumo do teste de F da Análise de Variância } \\
\hline$F_{\text {população }}(A)$ & 0,01 & $1 \mathrm{~ns}$ & & $1,41 \mathrm{~ns}$ & & & $1,90 \mathrm{~ns}$ & \\
\hline F cond.híd. (C) & 0,79 & 9 ns & & $7,95 *$ & & & $4,98 *$ & \\
\hline$F(A) \times F(C)$ & 1,00 & ns & & $2,90 \mathrm{~ns}$ & & & 3,03 ns & \\
\hline
\end{tabular}

${ }^{1}$ Médias não seguida pela mesma letra, maiúscula na coluna e minúscula na linha, diferem entre si pelo teste de Scott-Knott, em 5\% de probabilidade. COX: Coxilha; VAR: Várzea. CRA: capacidade de retenção de água do solo; LAM: Lâmina d'água. * significância em 5\%. ns: não significativo em nível de $5 \%$ de probabilidade de erro.

$\mathrm{Na}$ condição de déficit hídrico simulada no tratamento de $50 \%$ da CRA do solo as plantas de Várzea apresentaram maior eficiência no uso da água e maior eficiência instantânea de carboxilação 24 horas após o início dos tratamentos. Entretanto esta diferença não se manteve na avaliação de 192 horas (Tabela 5). Por outro lado, a população de Coxilha acumulou maior quantidade de CO2 intercelular se comparada à população de terras baixas. Para as demais variáveis aferidas atreladas ao comportamento fotossintético, não houveram diferenças significativas entre as populações na condição de 50\% da CRA do solo. As plantas 
C4, como o papuã, apresentam uma menor flutuação em seu desempenho fotossintético em condição de déficit hídrico, fixando com maior eficiência o carbono ao redor da enzima rubisco (TAYLOR et al., 2014). No tratamento de 100\% da CRA do solo, as plantas das populações de Coxilha e Várzea não diferiram estatisticamente para as variáveis relacionadas à fotossíntese, tanto na avaliação de 24 horas quanto na de 192 horas.

Tabela 5 - Médias da eficiência do uso da água (EUA - mol de CO2 mol H2O-1) e eficiência instantânea de carboxilação pela rubisco ( $\mathrm{A} / \mathrm{Ci}$ - $\mu \mathrm{mol} \mathrm{m}-2 \mathrm{~s}-1 \mathrm{~Pa}-1)$ em duas populações de plantas de Urochloa plantaginea 192 horas após o início dos tratamentos de condições hídricas do solo e resumo do teste de $\mathrm{F}$ da Análise de Variância

\begin{tabular}{|c|c|c|c|c|}
\hline \multirow{3}{*}{ Condição Hídrica } & \multicolumn{2}{|c|}{ EUA } & \multicolumn{2}{|c|}{ A/Ci } \\
\hline & \multicolumn{4}{|c|}{ População } \\
\hline & $\operatorname{cox}$ & VAR & $\operatorname{cox}$ & VAR \\
\hline $50 \% C R A$ & $6,28 \mathrm{~A} \mathrm{a}$ & $6,82 \mathrm{~A} \mathrm{a}$ & $0,11 \mathrm{~ns}$ & $0,12 \mathrm{~ns}$ \\
\hline $100 \% C R A$ & $6,98 \mathrm{~A} \mathrm{a}$ & $7,57 \mathrm{~A} \mathrm{a}$ & 0,19 & 0,15 \\
\hline$L A M$ & $5,24 \mathrm{~A} \mathrm{~b}$ & $6,79 \mathrm{~A} \mathrm{a}$ & 0,12 & 0,17 \\
\hline Média & $6,17 \mathrm{~b}$ & $7,06 \mathrm{a}$ & - & - \\
\hline CV (\%) & \multicolumn{2}{|c|}{14,38} & \multicolumn{2}{|c|}{45,48} \\
\hline \multicolumn{5}{|c|}{ Resumo do teste de F da Análise de Variância } \\
\hline$F_{\text {população }}(A)$ & \multicolumn{2}{|c|}{$5,29 *$} & \multicolumn{2}{|c|}{$0,15 \mathrm{~ns}$} \\
\hline $\mathrm{F}_{\text {cond.híd. }}$ (C) & \multicolumn{2}{|c|}{$3,53 \mathrm{~ns}$} & \multicolumn{2}{|c|}{$1,41 \mathrm{~ns}$} \\
\hline$F(A) \times F(C)$ & \multicolumn{2}{|c|}{$0,72 \mathrm{~ns}$} & \multicolumn{2}{|c|}{$0,78 \mathrm{~ns}$} \\
\hline
\end{tabular}

Médias não seguida pela mesma letra, maiúscula na coluna e minúscula na linha, diferem entre si pelo teste de Scott-Knott, em 5\% de probabilidade. COX: Coxilha; VAR: Várzea. CRA: capacidade de retenção de água do solo; LAM: Lâmina d'água. * significância em 5\%. ns: não significativo em nível de 5\% de probabilidade de erro.

Na coleta de dados realizada 24 horas após o início dos tratamentos, não houve diferença estatística para as variáveis A, Gs, Ci, E, EUA e A/Ci na condição hídrica de alagamento do solo (LAM). Entretanto, na avaliação de 192 horas, a população de Coxilha acumulou uma maior concentração de $\mathrm{CO}_{2}$ intercelular, obteve uma maior taxa de transpiração e condutância estomática em condição de lâmina d'água no solo. A população de Várzea por outro lado, acumulou menor 
quantidade de $\mathrm{CO}_{2}$ intercelular, apresentou uma menor taxa de transpiração e condutância estomática. As duas populações diferiram significativamente quanto à eficiência no uso da água, onde a população de terras baixas foi mais eficiente. Uma menor taxa de transpiração associada a uma maior eficiência no uso da água e a inexistência de diferença na taxa fotossintética entre as populações, indica um melhor controle no status hídrico das plantas de várzea em condição de lâmina d'água (MOLLARD et al., 2008). Além disso, as plantas que apresentam rota fotossintética C4 possuem uma menor variação em sua resposta da taxa fotossintética quando expostas a situações de estresse hídrico, devido a sua alta eficiência na fixação de $\mathrm{CO}_{2}$ (SAGE, 2000).

Analisando-se os parâmetros morfológicos pode-se inferir que a partir da média geral das populações de Várzea e Coxilha nos três ambientes, não houve diferença significativa para as variáveis massa seca de raiz (MSR), massa seca da parte aérea (MSPA), altura final da planta mãe (AFPM) e número total de perfilhos (NTP) ao fim do ciclo. As plantas de papuã das populações analisadas neste estudo responderam de maneira semelhante nas três condições hídricas do solo para a MSR e MSPA, considerando as médias gerais de cada condição hídrica (Tabela 6). Para a condição de lâmina d'água, as massas de raiz e parte aérea reduziram drasticamente quando comparadas com as condições de 50\% e 100\% da CRA do solo. Torna-se comum em plantas que não são oriundas de ambientes com elevada umidade do solo, a redução da massa seca quando expostas à esta condição hídrica (GOMATHI et al., 2010). Cabe ressaltar que para a variável MSPA, a população de $U$. plantaginea oriunda da Várzea demonstrou maior crescimento $(39,1 \mathrm{~g})$ em ambiente alagado, diferindo significativamente das plantas de Coxilha $(12,4$ g) nesta condição (Tabela 6). Em contrapartida, as plantas de Coxilha demonstraram uma resposta superior para MSPA em uma condição hídrica ótima (100\% da CRA do solo). Para Panicum virgatum L. identificou-se um ecótipo de terras baixas na qual não apresentava diferenças no crescimento tanto em ambiente 
alagado quanto em $100 \%$ CRA do solo, ao contrário do ecótipo de terras altas (BARNEY et al., 2009).

Tabela 6 - Médias da massa seca de raiz (MSR), massa seca da parte aérea (MSPA) de diferentes populações de Urochloa plantaginea, sob diferentes condições hídricas do solo

\begin{tabular}{|c|c|c|c|c|c|c|}
\hline \multirow{3}{*}{ Condição Hídrica } & \multicolumn{2}{|l|}{ MSR (g) } & \multicolumn{4}{|c|}{ MSPA (g) } \\
\hline & \multicolumn{6}{|c|}{ População } \\
\hline & $\operatorname{cox}$ & VAR & Média & $\operatorname{cox}$ & VAR & Média \\
\hline $50 \% C R A$ & $146,1 \mathrm{~A} \mathrm{a}$ & $141,5 \mathrm{~A} a$ & $\begin{array}{c}143,85 \\
\text { A }\end{array}$ & $80,8 \mathrm{~A} a$ & $81,7 \mathrm{~A} \mathrm{a}$ & $81,27 \mathrm{~A}$ \\
\hline $100 \% C R A$ & $155,8 \mathrm{~A} a$ & $155,8 \mathrm{~A}$ a & $\begin{array}{c}155,87 \\
A\end{array}$ & $88,6 \mathrm{~A} a$ & 73,8 A b & $81,22 \mathrm{~A}$ \\
\hline$L A M$ & $6,3 \mathrm{~B} \mathrm{a}$ & $16,8 \mathrm{~B} \mathrm{a}$ & $\begin{array}{l}11,60 \\
B\end{array}$ & $12,4 \mathrm{~B} \mathrm{~b}$ & $39,1 \mathrm{~B} \mathrm{a}$ & $25,81 \mathrm{~B}$ \\
\hline
\end{tabular}

CV (\%)

35,82

15,76

Resumo do teste de F da Análise de Variância

\begin{tabular}{|c|c|c|}
\hline$F_{\text {população }}(A)$ & $0,02 \mathrm{~ns}$ & $1,39 \mathrm{~ns}$ \\
\hline 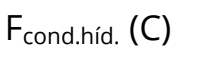 & $46,36 *$ & $104,74 *$ \\
\hline$F(A) \times F(C)$ & $0,11 \mathrm{~ns}$ & 11,24 \\
\hline
\end{tabular}

Médias não seguida pela mesma letra, maiúscula na coluna e minúscula na linha, diferem entre si pelo teste de Scott-Knott, em 5\% de probabilidade. COX: Coxilha; VAR: Várzea. CRA: capacidade de retenção de água do solo; LAM: Lâmina d'água. Significância em $5 \%$. ns: não significativo em nível de $5 \%$ de probabilidade de erro.

A população de Várzea $(94,0 \mathrm{~cm})$ quando submetida a condição de alagamento do solo, apresentou um maior crescimento médio em altura perante a população de Coxilha $(56,2 \mathrm{~cm}$ ) (Tabela 7). Nas espécies ambientadas à condição de lâmina d'água, observa-se uma maior altura, como exemplo do arroz, onde o acumulo de etileno induz a expressão dos genes SNORKEL1 e SNORKEL2, que via giberelina ocasionam em elongação dos entrenós (HATTORI et al., 2009). Já na condição ótima de disponibilidade de água para o papuã (100\% da CRA do solo), as plantas de Coxilha obtiveram um maior proveito desta condição no parâmetro em questão. Avaliando a resposta da população dentro de cada nível da condição hídrica do solo para a variável AFPM, notam-se algumas peculiaridades. A população de terras altas não diferiu em altura nas condições de $50 \%$ e $100 \%$ da 
CRA do solo. Sendo que, a de Várzea não diferiu em altura nas condições de lâmina d'água e $100 \%$ da CRA do solo. O que evidencia um comportamento diferencial das duas populações para a variável em questão nas condições impostas.

Tabela 7 - Médias da altura final da planta mãe (AFPM) e número total de perfilhos (NTP) de diferentes populações de Urochloa plantaginea, sob diferentes condições hídricas do solo

\begin{tabular}{|c|c|c|c|c|c|c|}
\hline \multirow{3}{*}{ Condição Hídrica } & \multicolumn{3}{|c|}{ AFPM (cm) } & \multicolumn{3}{|c|}{ NTP } \\
\hline & \multicolumn{6}{|c|}{ População } \\
\hline & $\operatorname{cox}$ & VAR & Média & $\operatorname{cox}$ & VAR & Média \\
\hline $50 \% C R A$ & $119,4 \mathrm{~A} a$ & $129,10 \mathrm{~A} \mathrm{a}$ & $124,25 \mathrm{~A}$ & $30,6 \mathrm{~A} \mathrm{a}$ & $22,6 \mathrm{~B} \mathrm{~b}$ & $26,60 \mathrm{~A}$ \\
\hline $100 \% C R A$ & $138,1 \mathrm{~A} \mathrm{a}$ & $98,80 \mathrm{~B} \mathrm{~b}$ & $118,45 \mathrm{~A}$ & $28,4 \mathrm{~A} a$ & $34,4 \mathrm{Aa}$ & $31,40 \mathrm{~A}$ \\
\hline$L A M$ & $56,2 \mathrm{~B} \mathrm{~b}$ & $94,00 \mathrm{~B} \mathrm{a}$ & $75,10 \mathrm{~B}$ & $5,6 \mathrm{~B} \mathrm{a}$ & $11,2 \mathrm{Ca}$ & $8,40 \mathrm{~B}$ \\
\hline CV (\%) & \multicolumn{3}{|c|}{14,03} & & 23,09 & \\
\hline \multicolumn{7}{|c|}{ Resumo do teste de F da Análise de Variância } \\
\hline $\mathrm{F}_{\text {população }}(\mathrm{A})$ & \multicolumn{3}{|c|}{$0,25 \mathrm{~ns}$} & \multicolumn{3}{|c|}{$0,41 \mathrm{~ns}$} \\
\hline Fond.híd. (C) & \multicolumn{3}{|c|}{$32,65^{*}$} & \multicolumn{3}{|c|}{$56,36 *$} \\
\hline$F(A) \times F(C)$ & \multicolumn{3}{|c|}{$17,22^{*}$} & \multicolumn{3}{|c|}{$6,08^{*}$} \\
\hline
\end{tabular}

Médias não seguida pela mesma letra, maiúscula na coluna e minúscula na linha, diferem entre si pelo teste de Scott-Knott, em 5\% de probabilidade. COX: Coxilha; VAR: Várzea. CRA: capacidade de retenção de água do solo; LAM: Lâmina d'água. Significância em 5\%. ns: não significativo em nível de 5\% de probabilidade de erro.

Em relação ao número total de perfilhos, as plantas de terras altas diferiram dos indivíduos de terras baixas somente na condição de déficit hídrico (50\% da CRA), caracterizando-se por demonstrarem uma maior capacidade de perfilhamento nesta condição (Tabela 7). Nas demais condições hídricas, as populações não se diferenciaram estatisticamente em NTP. Para a população de Coxilha, não houve distinção no número de perfilhos nas condições de 50\% e 100\% da CRA, porém na condição de lâmina d'água a emissão de perfilhos foi reduzida. Mesmo gramíneas que habitam solos com frequente déficit hídrico podem reduzir o número de perfilhos nesta condição devido a menor disponibilidade do recurso, fato este que não foi demonstrado pela população de Coxilha (NEZAR, 2005). Em contrapartida, para as plantas de Várzea, a resposta aos níveis de umidade do solo 
foi outra, onde a condição de déficit hídrico reduziu o NTP quando comparada a condição ótima de água no solo (100\% da CRA). Em plantas ambientadas a condição de alagamento, quando expostas à condição de déficit hídrico naturalmente tendem a reduzir o número de perfilhos e sua altura (FAROOQ et al., 2010).

Outro parâmetro avaliado foi a peroxidação de lipídeos tanto em raizes quanto na parte aérea das plantas, 24 horas e 192 horas após o início dos tratamentos de condição hídrica do solo. Em raízes, não houveram diferenças estatísticas nos fatores avaliados em nenhum dos momentos de coleta dos dados (Tabela 8).

Tabela 8 - Médias da peroxidação de lipídeos (nmol de MDA mg ${ }^{-1}$ de proteína) radicular, 24 e 192 horas após o início dos tratamentos em duas populações de plantas de Urochloa plantaginea sob diferentes condições hídricas do solo e resumo do teste de $\mathrm{F}$ da Análise de Variância

\begin{tabular}{|c|c|c|c|c|}
\hline \multirow{3}{*}{ Condição Hídrica } & \multicolumn{2}{|c|}{$24 \mathrm{~h}$} & \multicolumn{2}{|c|}{$192 \mathrm{~h}$} \\
\hline & \multicolumn{4}{|c|}{ População } \\
\hline & $\operatorname{cox}$ & VAR & $\operatorname{cox}$ & VAR \\
\hline $50 \% C R A$ & $0,0045 \mathrm{~ns}$ & $0,0105 \mathrm{~ns}$ & $0,0084 \mathrm{~ns}$ & $0,0036 \mathrm{~ns}$ \\
\hline $100 \% C R A$ & 0,0048 & 0,0056 & 0,0116 & 0,0075 \\
\hline$L A M$ & 0,0065 & 0,0108 & 0,0027 & 0,0062 \\
\hline \multirow[t]{2}{*}{ CV (\%) } & \multicolumn{2}{|c|}{30,21} & \multicolumn{2}{|c|}{28,24} \\
\hline & \multicolumn{4}{|c|}{ Resumo do teste de F da Análise de Variância } \\
\hline$F_{\text {população }}(A)$ & \multicolumn{2}{|c|}{$2,45 \mathrm{~ns}$} & \multicolumn{2}{|c|}{$0,79 \mathrm{~ns}$} \\
\hline 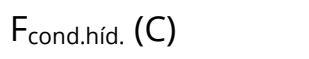 & \multicolumn{2}{|c|}{$0,76 \mathrm{~ns}$} & \multicolumn{2}{|c|}{$2,23 \mathrm{~ns}$} \\
\hline$F(A) \times F(C)$ & \multicolumn{2}{|c|}{0,43 ns } & \multicolumn{2}{|c|}{$1,74 \mathrm{~ns}$} \\
\hline
\end{tabular}

Médias não seguida pela mesma letra, maiúscula na coluna e minúscula na linha, diferem entre si pelo teste de Scott-Knott, em 5\% de probabilidade. COX: Coxilha; VAR: Várzea. CRA: capacidade de retenção de água do solo; LAM: Lâmina d'água. * significância em 5\%. ns: não significativo em nível de 5\% de probabilidade de erro.

Tratando-se da peroxidação lipídica de parte aérea, não se evidenciou diferenças significativas em nenhum dos fatores no período de 24 horas após o início dos tratamentos (Tabela 9). Entretanto, na avaliação 192 horas após o início dos tratamentos de umidade do solo observou-se uma interação entre os fatores 
população e condição hídrica do solo significativa. As plantas de papuã originárias de Coxilha apresentaram uma maior peroxidação de lipídeos nas situações de estresse tanto por falta quanto por excesso d'água, diferindo significativamente da condição de $100 \%$ da CRA do solo. As plantas de Várzea não diferiram estatisticamente para a peroxidação de lipídeos nos três diferentes ambientes, indicando uma melhor resposta das plantas ao estresse hídrico em ambas as condições.

Tabela 9 - Médias da peroxidação de lipídeos (nmol de MDA mg-1 de proteína) em parte aérea 24 e 192 horas após o início dos tratamentos em duas populações de plantas de Urochloa plantaginea, sob diferentes condições hídricas do solo e resumo do teste de F da Análise de Variância

\begin{tabular}{|c|c|c|c|c|c|}
\hline \multirow{3}{*}{ Condição Hídrica } & \multicolumn{2}{|c|}{$24 \mathrm{~h}$} & \multicolumn{3}{|c|}{$192 \mathrm{~h}$} \\
\hline & \multicolumn{5}{|c|}{ População } \\
\hline & $\operatorname{cox}$ & VAR & $\operatorname{cox}$ & VAR & Média \\
\hline $50 \% C R A$ & $0,0165 \mathrm{~ns}$ & $0,0153 \mathrm{~ns}$ & $0,0157 \mathrm{~A} \mathrm{a}$ & $0,0150 \mathrm{~A} \mathrm{a}$ & $0,0153 \mathrm{~A}$ \\
\hline $100 \% C R A$ & 0,0168 & 0,0155 & $0,0120 \mathrm{~B}$ a & $0,0122 \mathrm{~A} \mathrm{a}$ & $0,0121 B$ \\
\hline$L A M$ & 0,0170 & 0,0164 & $0,0178 \mathrm{~A} \mathrm{a}$ & $0,0135 \mathrm{~A} \mathrm{~b}$ & $0,0156 \mathrm{~A}$ \\
\hline CV (\%) & \multicolumn{2}{|c|}{20,12} & \multicolumn{3}{|c|}{14,78} \\
\hline \multicolumn{6}{|c|}{ Resumo do teste de F da Análise de Variância } \\
\hline$F_{\text {população }}(A)$ & \multicolumn{2}{|c|}{$0,60 \mathrm{~ns}$} & \multicolumn{3}{|c|}{3,33 ns } \\
\hline 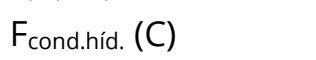 & \multicolumn{2}{|c|}{$0,119 \mathrm{~ns}$} & \multicolumn{3}{|c|}{$6,75^{*}$} \\
\hline$F(A) \times F(C)$ & \multicolumn{2}{|c|}{$0,21 \mathrm{~ns}$} & \multicolumn{3}{|c|}{$2,47 \mathrm{~ns}$} \\
\hline
\end{tabular}

Médias não seguida pela mesma letra, maiúscula na coluna e minúscula na linha, diferem entre si pelo teste de Scott-Knott, em 5\% de probabilidade. COX: Coxilha; VAR: Várzea. CRA: capacidade de retenção de água do solo; LAM: Lâmina d'água. * significância em 5\%. resumo do teste de $\mathrm{F}$ da Análise de Variância. ns: não significativo em nível de $5 \%$ de probabilidade de erro.

A análise de peroxidação de lipídeos é um bom indicador para avaliar a sensibilidade de uma planta à uma condição de estresse (HERZOG et al., 2016). Em plantas expostas a condições hipóxicas e anóxicas, Li et al. (2006) relataram que ocorrem extensas peroxidações de lipídeos, sendo relacionado à peroxidação de membranas. Esta degradação de tecidos internos é justificada em condições hipóxicas devido ao acúmulo de espécies reativas de oxigênio (EROs) na parte 
aérea de gramíneas (GOMATHI; CHANDRAN, 2012). Observando o fator condição hídrica do solo dentro de cada população, ressalta-se a maior peroxidação de lipídeos na população de Coxilha e menor na população de Várzea sob condição de lâmina d'água. Após 192h do alagamento observou-se uma maior redução na massa seca e uma maior peroxidação de lipídeos nas duas populações de Urochloa plantaginea. Em condição de lâmina d'água no solo a alta redução de massa seca em parte aérea comumente é relacionada à alta peroxidação de lipídeos (SHAO et al., 2013). Normalmente as respostas de variáveis relacionadas a peroxidação de lipídeos ocorrem cerca de 4 a 6 dias após a exposição à condição hídrica, explicando a significância dos resultados 192h após o ínicio dos tratamentos de umidade do solo (TANG et al., 2010; HERZOG et al., 2016).

As plantas oriundas de um ambiente de várzea quando comparadas às plantas de coxilha, demonstraram no presente estudo características morfofisiológicas superiores em condição de alagamento do solo. Pode-se ressaltar, maior eficiência no uso da água (cerca de 30\%), maior altura de plantas, menor peroxidação de lipídeos de parte aérea e um maior incremento de massa seca de parte aérea. Adicionalmente, a população de várzea demonstrou não variar os parâmetros fotossintéticos A, Gs, Ci, E, EUA e A/Ci quando exposta à lâmina d'água, isto comparando-se com a condição de $100 \%$ da CRA. O comportamento desta população em ambiente alagado demonstra uma maior adaptação ao mesmo, evidenciando características para superar as limitações impostas pelo ambiente hipóxico/anóxico.

\section{CONCLUSÕES}

Os resultados apresentados neste trabalho permitem concluir que a condição hídrica do solo é limitante para o crescimento e desenvolvimento de Urochloa plantaginea. As diferentes disponibilidades de água no solo impostas às plantas, possibilitaram caracterizar as respostas de cada população frente a estes 
ambientes. Ambas as populações suportaram o alagamento até o final do ciclo e produziram sementes, além disso não se diferenciaram em $\mathrm{A}, \mathrm{A} / \mathrm{Ci}$ (192h), número total de perfilhos, MSR e peroxidação de lipídeos radicular (24h e 192h).

\section{REFERÊNCIAS}

ARMSTRONG W, BRANDLE R, JACKSON MB. Mechanisms of flood tolerance in plants. Acta Botanica, v. 43, n. 4, p. 307-358, 1994.

BARNEY JN, MANN JJ, KYSER GB, BLUMWALD E, DEYNZE AV, DITOMAZO JM. Tolerance of switchgrass to extreme soil moistures stress: Ecological implications. Plant Science, v. 177, n. 6, p. 724-732, 2009. DOI: 10.1016/j.plantsci.2009.09.003

BIANCHI MA. Programa de difusão de manejo integrado de plantas daninhas em soja. In: Reunião de pequisa de soja da Região Sul, 23., 1996, Porto Alegre. Ata e Resumos. Porto Alegre: Universidade Federal do Rio Grande do Sul, 1996. p. 125.

BOLDRINI II, LONGHI-WAGNER HM, BOECHAT SC. Morfologia e taxonomia de Gramíneas Sulrio-grandenses. 2 ed. Porto Alegre: Editora da Universidade/UFRGS, 2008. 87 p.

BRAMLEY H, TYERMAN SD. Root water transport under waterlogged conditions and the roles of aquaporins. In: Waterlogging Signalling and Tolerance in Plants (eds Mancuso S. \& Shabala S.). Springer, Berlin Heidelberg, p. 151-180, 2010.

EL-MOSHATY FIB, PIKE SM, NOVACKY AJ, SEHGAL OP. Lipid peroxidation and superoxide productions in cowpea (Vigna unguiculata) leaves infected with tobacco rings virus or southern bean mosaic virus. J. Physiol Mol. Plant Pathol., v. 43, p. 109-119, 1993.

ESTATCAMP. Software Action. Estatcamp - Consultoria em estatística e qualidade, São Carlos, SP, 2014. Disponível em: http://www.portalaction.com.br Acesso em: 27 jun. 2016.

FAROOQ M, KOBAYASHI N, ITO O, WAHID A, SERRAJ R. Broader leaves result in better performance of indica rice under drought stress. Journal of Plant Physiology, v. 13, p. 10661075, 2010. DOI: 10.1016/j.jplph.2010.03.003.

FERREIRA DF. Sisvar: a computer statistical analysis system. Ciência e Agrotecnologia (UFLA), v. 35, n. 6, p. 1039-1042, 2011.

GOMATHI R, CHANDRAN KPN, GURURAJA R, RAKKIYAPPAN P. Effect of waterlogging in sugarcane and its management. Sugarcane Breeding Institute (SBI-ICAR), Extension Pub. $\mathrm{n}$. 185, 2010. 
GOMATHI R, CHANDRAN K. Physiological markers for screening waterlogging Resistance in sugarcane. In: Proceedings of International symposium on "New Paradigms in Sugarcane Research" ISNPSR 2012. Organised by SSRD \& SBI at Coimbatore. Abstract No. 129, 2012.

HATTORI Y, NAGAI K, FURUKAWA S, SONG XJ, KAWANO R, SAKAKIBARA H, MATSUMOTO T, YOSHIMURA A, KITANO $H$. The ethylene response factors SNORKEL1 and SNORKEL2 allow rice to adapt to deep water. Nature, v. 460, p. 1026-1030, 2009. DOI:10.1038/nature08258

HERZOG M, STRIKER GG, COLMER TD, PEDERSEN O. Mechanisms of waterlogging tolerance in wheat - a review of root and shoot physiology. Plant, Cell and Environment, v. 39, p. 10681086, 2016. DOI: 10.1111/pce.12676

KISSMANN KG. Plantas infestantes e nocivas. 2. ed., Tomo I, São Paulo: Basf S.A., CD-ROOM. 1997.

KOZLOWSKI TT. Plant responses to flooding of soil. Bioscience, v. 34, p. 162-167, 1984.

KRAEHMER H, BAUR P. Weed Anatomy. Wiley-Blackwell, Chichester, UK. 2013

LI JC, WEI FZ, WANG CY, YIN J. Effects of waterlogging on senescence of root system at booting stage in winter wheat. Acta Agronomica Sinica, v. 9, p. 1355-1360, 2006.

LORENZI H. Plantas daninhas do Brasil: terrestres, aquáticas, parasitas e tóxicas. 3.ed. Nova Odessa: Plantarum, 2000. 608 p.

MACEDO LCP. Alterações morfológicas em plantas do gênero Urochloa P. Beauv. submetidas a três condições de umidade do solo. 2015. 69 f. Dissertação de Mestrado (Mestrado em Agrobiologia)-Universidade Federal de Santa Maria, Santa Maria, 2015.

MOLLARD FPO, STRIKER GG, PLOSCHUK EL, VEGA AS, NSAUSTI P. Flooding tolerance os Paspalum dilatatum (Poaceae: Paniceae) from upland and lowland positions in natural grassland. Flora, v. 203, p. 548-556, 2008. DOI: 10.1016/j.flora.2007.10.003

MOREIRA HJC, BRAGANÇA HBN. Manual de Plantas Infestantes: Arroz. São Paulo: Ed. FMC Agricultural Products. 2010.642 p.

NEZAR SH. Effects of drought stress on growth and yield of barley. Agronomy for Sustainable Development, v. 25, n. 1, p. 145-149, 2005. DOI: 10.1051/agro:2004064

PEZESHKI SR. Wetland plant responses to soil flooding. Environmental and Experimental Botany, v. 46, p. 299-312, 2001. DOI: 10.1016/S0098-8472(01)00107-1

SAGE RF. C3 versus C4 photosynthesis in rice: ecophysiological perspectives. Studies in Plant Science. v. 7, p. 13-25, 2000. DOI: 10.1016/S0928-3420(00)80004-X 
SHAO GC, LAN JJ, YU SE, LIU N, GUO RQ, SHE DL. Photosynthesis and growth of winter wheat in response to waterlogging at different growth stages. Photosynthetica, v. 51, n. 3, p. 429-437, 2013. DOI: 10.1007/s11099-013-0039-9

TANG B, XU S, ZOU X, ZHENG Y, QIU F. Changes of Antioxidative Enzymes and Lipid Peroxidation in Leaves and Roots of Waterlogging-Tolerant and Waterlogging-Sensitive Maize Genotypes at Seedling Stage. Agricultural Sciences in China, v. 9, n. 5, p. 651-661, 2010. DOI: 10.1016/S16712927(09)60140-1

TAYLOR SH, RIPLEY BS, MARTIN T, DE-WET L, WOODWARD FI, OSBORNE CP. Physiological davantages of $\mathrm{C} 4$ grasses in the field: a comparative experimente demonstrating the importance of drought. Global Change Biology, v. 20, p. 1992-2003, 2014. DOI: $10.1111 /$ gcb. 12498

VELHO GF, CRUSCIOL CAC, VELINI ED, CASTRO GSA, BORGHI E. Interferência de Brachiaria plantaginea com a cultura do arroz, cv. Primavera. Planta Daninha, v. 30, n. 1, p. 17-26, 2012. DOI: $10.1590 /$ S0100-83582012000100003

WANG Q, CHEN J, LIU F. Morphological changes and resource allocation of Zizania latifolia (Griseb.) Stapf in response to different submergence depth and duration. Flora, v. 209, p. 279284, 2014. DOI: 10.1016/j.flora.2014.03.006

\section{Contribuição dos Autores}

\section{1 - Leandro Lima Spatt}

Engenheiro Agrônomo

https://orcid.org/0000-0003-1108-6471 - leandrolimaspatt@gmail.com

Contribuição: Validação

\section{2 - Sidinei José Lopes}

Doutorado em Agronomia

https://orcid.org/0000-0002-7117-541X - sjlopes2008@gmail.com

Contribuição: Conceituação

\section{3 - Sylvio Henrique Bidel Dornelles}

Doutorado em Agronomia

https://orcid.org/0000-0002-1097-6176 - sylviobidel@gmail.com

Contribuição: Conceituação

\section{4 - Vinicius Severo Trivisiol}

Doutorado em Agronomia https://orcid.org/0000-0003-1108-6471 - vinicius_trivisiol@hotmail.com Contribuição: Validação; investigação 


\section{5 - Mariane Peripolli}

Doutorado em Agronomia

https://orcid.org/0000-0002-2147-9458 - mperipolli@gmail.com

Contribuição: Validação

\section{6 - Elisandro Azeredo Nunes}

Graduação em Agronomia

https://orcid.org/0000-0002-5780-4478 e elisandroeng.agro@gmail.com

Contribuição: Investigação 